Aims. To determine compliance with the new discharge policy of review within 7-days for all General Adult Psychiatry patients discharged from Forth Valley Royal Hospital.

Background. It is well established that there is an increased risk of suicide following discharge from Inpatient Psychiatric Wards. This risk is significantly increased in the first month, and particularly high in the first week.

In their 2016 Guidance, NICE recommends follow-up within 7 days of discharge. It is not known whether seven day follow-up reduces suicide risk but it is clearly an opportunity for risk assessment and management during a particularly risky period.

This standard was adopted by the General Adult Wards in Mental Health Unit at Forth Valley Royal Hospital in April 2019. Method. All discharges from Wards 1, 2 and 3, Forth Valley Royal Hospital were reviewed during three distinct, month-long periods:

November 2018 (prior to the introduction of the new discharge policy)

May 2019 (shortly after the introduction of the new discharge policy)

September 2019 (six months after the introduction of the new discharge policy)

A list was obtained from Medical Records of all General Adult patients discharged in these periods. The paper and electronic records were checked for each patient, and the first scheduled care episode post discharge was taken as follow-up.

Result. In the1st round of audit (November 2018): 41 patients were discharged and 26 patients (64\%) received follow-up within 7 days.

In the 2nd round of audit (May 2019): 46 patients were discharged, 39 patients (84\%) received follow-up within 7 days.

In the 3rd round of the audit (September 2019), 50 patients were discharged and 49 (98\%) received follow-up within 7 days. Conclusion. There has been a clear improvement in the provision of follow-up on discharge from the General Adult Psychiatry Wards in Forth Valley Royal Hospital.

The new discharge policy was implemented in April 2019 and a "Discharge Pause" was introduced (initially a sticker, now an electronic form) to be completed by the medical team at the point when it was decided to discharge.

Community Mental Health Teams have also been reminded of their need to facilitate seven day follow-up as a priority. A flowchart was produced in May 2019, which provided guidance as to who should provide the seven day follow-up.

\section{Professional training and case-load mix within a community mental health team}

Richard Walsh ${ }^{1 \star}$, Rebecca Fahy ${ }^{2}$, Ala Abdelgadir ${ }^{3}$, Elizabeth Walsh ${ }^{3}$ and Sonn Patel ${ }^{3}$

${ }^{1}$ School of Medicine, University College Dublin; ${ }^{2}$ Semmelweis University and ${ }^{3}$ Galway University Hospital

${ }^{\star}$ Corresponding author.

doi: 10.1192/bjo.2021.603

Aims. Community Mental Health Teams (CMHTS) are now the cornerstone of modern mental health care and play a central role in assessment, diagnosis and care coordination. CMHTs vary widely in their service provision and composition. Within teams there is latitude for variation of professional roles but the extent to which different disciplines undertake generic and profession-specific work is poorly defined. This cross-sectional study aims to establish how professional training influences the distribution of case-load mix within a general adult CMHT
Method. The GR1 CMHT provides care to a mixed urban/rural population of 25,000 in Galway city and Connemara. A review was conducted of multi-disciplinary case notes for all patients actively registered with the team for a period of one year. Name, age, gender, whether referred or admitted in the past year, medication and day hospital attendance were recorded. Clinical diagnoses were recorded but, where missing, verified with a relevant team member. The team consultant reviewed and verified the $1 \mathrm{CD}-10$ primary clinical diagnosis for all patients. Evidence of clinical input by multidisciplinary team members was recorded from clinical files with the final electronic database being checked by each professional for accuracy. We examined any input over the past year rather than

frequency of input. Patient characteristics and diagnosis by professional discipline were examined using descriptive statistics. Result. Of a total of 246 patients registered to the team, $37.8 \%$ (N = 93) saw one, $34.6 \%(\mathrm{~N}=85)$ saw two and $24.4 \%(\mathrm{~N}=60)$ saw 3 or more team members. Of those who saw three or more team members, psychotic disorders represented the majority diagnoses $(40 \%, \mathrm{~N}=24)$ followed by personality disorders $(25 \%, \mathrm{~N}=15)$ and affective disorders $(15 \%, \mathrm{~N}=9)$. Patients were most commonly seen by a doctor $(91.5 \%, \mathrm{~N}=225)$ followed by community mental health nurses (CMHNs) $(52.8 \%, \mathrm{~N}=130)$. Doctors saw $85 \%$ or more of all patients grouped by ICD-10 diagnoses. The majority of social work and occupational therapy case-mix comprised psychotic disorders ( $\mathrm{SW}=44.2 \%$, OT $=34.2 \%$ ) followed by personality disorders $(\mathrm{SW}=25.6 \%$, OT $=23.7 \%)$. Of psychology case-mix, the highest was personality

disorders at $41.6 \%(\mathrm{~N}=13)$ followed by anxiety and related disorders at $25 \%(\mathrm{~N}=8)$. CMHN case-mix was highest for psychotic disorders at $44.6 \%(\mathrm{~N}=58)$ followed by $21.5 \% \operatorname{mood}$ disorders $(\mathrm{N}=28)$.

Conclusion. This cross sectional survey informs how we currently target our specialist resources. We will now develop this to include frequency of contact to inform resource allocation and skill mix.

\section{Mental health clothing bank - adDressing the issue Louisa Ward}

Worcestershire health and care NHS trust

doi: 10.1192/bjo.2021.604

Aims. We often have patients who are admitted to the ward wearing only the clothes they came in. These patients have no way of going to get more clothes due to being detained, poverty/ homelessness or covid restrictions. Many do not have friends or family who can bring them clothes. As such they might wear one set of clothes for a number of weeks which is bad for their physical and mental health. We are creating a clothes bank to provide a change of clothes for these patients, and help their recovery back into the community. Many have clothes that are inappropriate for the current weather, or do not have a set of smart enough clothes for a job interview. We feel that this simple intervention will have a big community impact.

Method. We have obtained support from a number of charities and companies to supply donations. The project will be led by a team of staff and patients.

Result. We will review the usage of this scheme in 6 months time Conclusion. We hope this intervention will tackle the issue of clothing on mental health wards. In the future we wish to expand this to outpatient mental health service users. We would then like to expand this project countrywide as are unaware of any other areas providing something similar. 


\section{Site downsizing: a blessing or a curse?}

Benjamin Waterhouse ${ }^{\star}$ and Ben Attwood

Camden \& Islington NHS Trust

${ }^{*}$ Corresponding author.

doi: 10.1192/bjo.2021.605

Aims. To measure staff wellbeing and morale, which in 2015 was described by CQC as 'low', following a downsizing of premises.

Background. In 2019, due to loss of mental health funding, Camden \& Islington NHS Foundation Trust controversially sold the much-loved Queen Anne-style mansion Lyndhurts Gardens. The Rehab \& Recovery team caring for those with serious mental illness were relocated to one floor of the much smaller Daleham Gardens. It was hypothesed by the authors that this would impact negatively on the already unhappy workforce.

Method. The same staff wellbeing survey was used as in in 2015 (following CQC's description of 'poor' morale), nearly 5 years on and following the site relocation. All clinical, managerial and administrative staff members were encouraged to participate by posting their survey anonymously in a make-shift postbox. Sweet treats were used to encourage participation within the busy team.

Result. Qualitative and quantitative data were collected from the team (response rate 44\%). Exact tables will be shown but in summary; in the new building there was an increase in the number of staff who felt they could use initiative at work, make improvements at work, looked forward to going into work and felt enthusiastic about their job. Improvement cited were 'increased socials after work' and consequent 'wellbeing', in addition to 'good team atmosphere', 'good team work' and 'good relationships' in the new space. Further ideas were generated for additional improvements going forward.

Conclusion. Whilst caseloads and workloads are often cited as the cause of burnout, and indeed other changes in the 5 years could act as confounders, the design of work buildings is not to be underestimated. Contrary to what the authors had suspected, 'bigger' was not necessarily 'better' and a more condensed working environment made for greater togetherness amongst the team. In this age where economically desperate NHS trusts are forced to sell off their prized assets, observations that this is not always at the detriment of staff morale will provide some cause for optimism.

\section{Views of medical educators on virtual teaching methods and curriculum changes within an undergraduate psychiatry rotation during the COVID-19 pandemic - a quality improvement project \\ Ellen Williams*, Nidhi Gupta and Femi Oyebode \\ Birmingham and Solihull Mental Health NHS Foundation Trust ${ }^{*}$ Corresponding author.}

doi: 10.1192/bjo.2021.606

Aims. Medical students at the University of Birmingham have historically undertaken a 9 week psychiatry rotation consisting of clinical placements accompanied by lectures and small group teaching. During the COVID-19 pandemic teaching has been offered in a virtual format and clinical placements have been restricted. Our aim was to survey medical educators regarding these changes, seeking their views on resources and skills required for virtual teaching, student engagement, and adaptations to placements.
Method. 73 medical educators who had undertaken teaching or clinical supervision were invited to complete an anonymous online survey during December 2020. The survey consisted of Likert scale and open space questions surrounding changes necessitated by the pandemic. Following survey closure quantitative data were analysed using Google Forms and Excel. Qualitative data from the survey were reviewed by all team members to identify relevant themes.

Result. Overall response rate was 40\% (29), with participants from 5 NHS trusts. $72 \%$ (21) of educators felt they had adequate equipment and resources to facilitate virtual teaching. 55\% (16) felt they had adequate training and skills to use virtual teaching platforms effectively. However only 17\% (5) felt that students were able to engage in virtual teaching to the same extent as face to face sessions, and just 35\% (10) of educators reported enjoying virtual teaching. $76 \%$ (22) agreed that information from the University about adjustments to clinical placements was adequate. $66 \%$ (19) felt that there had been adequate support to ensure the safety of students, supervisors and patients. However only $20 \%$ (6) felt that students had adequate patient contact and 69\% (20) did not feel that students had been able meet their clinical competencies.

Conclusion. Our results suggest that the majority of educators have not enjoyed teaching virtually, and feel that students were less engaged. However educators were able to identify some benefits, such as inviting speakers from outside the local area, improved access to manager's hearings and tribunals and the use of simulated patients. There were also innovative suggestions, such as interactive quizzes and feedback polls within sessions. Most educators felt students had not received adequate patient contact during the pandemic and suggestions for improvement were less readily identified, they included changes to work place based assessments and timetabling. We hope using these results to work with the University to develop resources to support educators using virtual teaching methods, and to consider adjustments to clinical placement while the pandemic persists.

\section{Improving physical health form completion on a general inpatient adult ward}

Brandon Wong* and Anjna Vekaria

CNWL

${ }^{\star}$ Corresponding author.

doi: 10.1192/bjo.2021.607

Aims. The government's Five Year Forward View Plan for Mental Health has set a target for 280,000 people with severe mental health problems to be offered screening and appropriate intervention based on physical health risk stratification, including obesity, diabetes and heart disease. As such, physical health review for patients on a general inpatient adult psychiatry ward includes routine blood tests for cholesterol levels and HbAlc. They are recorded together in a Physical Health $(\mathrm{PH})$ Form in the patient's electronic record and used to stratify cardiovascular risk factors and risk of diabetes. If a patient declines these blood tests it should be recorded on the $\mathrm{PH}$ form.

This study aims to improve the completion of Physical Health forms to $\geq 95 \%$ by within a 4 -month period on a general adult inpatient psychiatric ward.

Method. PH form completion was measured using Tableau Software for a 4 -week period as a baseline then fortnightly during the study. $\mathrm{PH}$ form completion required $\mathrm{HbAlc}$ and cholesterol levels to be inputted, or to be marked as declined where the patient had declined these tests. Potential interventions were 